Journal of Bioinformatics and Computational Biology

Vol. 11, No. 6 (2013) 1302003 (4 pages)

Imperial College Press

(C) Imperial College Press

DOI: $10.1142 /$ S0219720013020034

\title{
THE 24TH INTERNATIONAL CONFERENCE ON GENOME INFORMATICS, GIW2013, IN SINGAPORE
}

\author{
FRANK EISENHABER \\ Bioinformatics Institute, Agency for Science \\ Technology and Research, 30 Biopolis Street \#07-01 \\ Matrix, Singapore 138671 \\ Department of Biological Sciences \\ National University of Singapore \\ 8 Medical Drive, Singapore 117597 \\ School of Computer Engineering \\ Nanyang Technological University \\ 50 Nanyang Drive, Singapore 637553 \\ franke@bii.a-star.edu.sg \\ WING-KIN SUNG \\ School of Computing \\ National University of Singapore \\ 13 Computing Drive, Singapore 117417 \\ Genome Institute of Singapore \\ 60 Biopolis Street \#02-01, Genome \\ Singapore 138672 \\ ksung@comp.nus.edu.sg \\ LIMSOON WONG \\ School of Computing \\ National University of Singapore \\ 13 Computing Drive, Singapore 117417 \\ wongls@comp.nus.edu.sg \\ Accepted 26 November 2013 \\ Published 18 December 2013
}

The "International Conference on Genome Informatics", popularly known as "GIW", is probably one of the oldest, if not actually the oldest annual, regular conference in computational biology. This conference is surprisingly viable, it survived all turns of the tempestuous development of this field of research. ${ }^{1}$ It is impossible to overestimate the role of GIW in establishing and enhancing the computational biology 
and bioinformatics research community in the Asia-Pacific region and its interaction with the world-wide research effort. Importantly, it has provided a friendly forum where scientists especially from the region could exchange and publish their research findings. It has accompanied and furthered the growth of computational biology and bioinformatics research in both quantity and quality in the Asia-Pacific region.

The GIW was first held as an open workshop ("Genome Informatics Workshop", thus, GIW) at Kikai Shinko Kaikan in Tokyo during December 3-4, 1990, essentially just before the Japanese Human Genome Project started in the next year. Whereas GIW was originally an intra-Japanese affair, it changed to an international conference in 1993 and the currently used name of the conference was adopted in 2001. During the last $\sim 15$ years, the conference was always attended by several hundred participants; thus, it is not really a "workshop" any longer. Whereas GIW had more the role of a regional incubator in the early years, it has recently become one of the important, truly international conference venues in the bioinformatics field for scientific exchange. It provides unique opportunities to bridge theory and experiment, academia and industry, science from the East and the West.

The conference site was in Tokyo or Yokohama exclusively until 2006 (as well as 2009). GIW 2007 (the 18th edition) was the first one held outside Japan, in the Biopolis in Singapore. Other locations in the Asia-Pacific regions were to follow: the 19th GIW at the Gold Coast in Australia (2008), the 21st GIW in Hangzhou (China) in 2010, the 22nd GIW in Busan (South Korea) in 2011 and the 23rd GIW in Tainan (Taiwan).

Remarkably, the 24th GIW has been awarded to Singapore again ${ }^{2}$ and, notably, is held in the same premises as the conference in 2007, namely in the Matrix Building of Biopolis. Singaporean bioinformaticians might tend to see this as recognition for their research efforts during the last years; though, the geographically central location in the Asia-Pacific region, the excellent transport hub and the infrastructural support of Singapore will lend an alternative, equally important explanation. All events happen only if an activist champions them. The Singaporean community is grateful to Limsoon Wong for his lobbying effort to attract important conferences here.

Certainly, the maturity of the research area and today's scientific fashions affect the research efforts that occupy a prominent place in GIW 2013. New methodological developments in classical research areas such as biomolecular sequence analysis, 3D structural analysis and modeling, etc. still remain important areas for a continued scientific quest. It should be noted that it was the concept of sequence homology and the subsequent opportunity for function prediction of uncharacterized genes via annotation transfer that, historically for the first time, made theoretical biology and its more fashionable offshoots bioinformatics and computational biology center stage in life sciences for all branches that are concerned about biomolecular mechanisms. ${ }^{3-6}$

At the same time, the availability of large-scale experimental datasets from genome-wide sequencing, expression profiling, automated bioimaging, etc. dramatically enhances the role of bioinformatics becoming an integral part of standard life 
science research projects. In contract to previous eras where the actual measurement of quantified data about biological systems was the bottleneck, the shift is now towards interpretation of the data in biological and biomolecular mechanism terms. This trend potentially allows also for a more system-wide view of description and analysis.

Given the absence of a consistent, predictive biological theory as physicists have been used to since many decades, some might consider the request for an integrated, system approach grandiloquent and premature. There are serious arguments for this view as about $50 \%$ of all eukaryote genes lack even tentative functional characterizations and, most likely, not even half of the biomolecular mechanisms are known. ${ }^{7}$ Despite full genome sequencing, even a stable reference proteome cannot be deduced. ${ }^{8}$ Thus, quantitative and predictive biology has a long way to go.

Therefore, it might be a good idea to generate large-scale data about biological systems only with a concrete biological question in mind and to plan the process in cooperation with computational biologists from the very beginning. ${ }^{3}$ Unfortunately, many big sequencing datasets generated today and in the close past just for the purpose of having a public resource, probably, will have never been properly analyzed at all since neither the manpower capacity for their study had ever been allocated in the community nor these datasets will be needed in the future since they will be re-created for lower costs and with higher accuracy then.

Nevertheless, the large-scale experimental techniques, most prominently nucleic acid sequencing but also epigenetics analyses, large-scale expression studies, proteomics with the large sets of protein-protein interaction data, the ever growing library of biomacromolecular structures and automated methods for analyzing cellular and tissue images ${ }^{9}$ open new opportunities and, for carefully selected questions, interesting and important insights can be deduced from this data at the biological systems level that can even reach out into biomedical and biotechnological applications. The papers from GIW 2013 collected in this special edition, as well as two supplementary volumes of BMC Systems Biology (Vol. 7, Suppl. 6) and ACM/IEEE Transactions on Bioinformatics and Computational Biology, exemplify how far research has moved forward.

\section{References}

1. GIW International Conference on Genome Informatics 1990-2009 [http://giw.hgc.jp].

2. Welcome to the 24th International Conference on Genome Informatics GIW 2013 [http:// www.comp.nus.edu.sg/ giw2013/].

3. Ouzounis CA, Two or three myths about bioinformatics, Bioinformtics 16:187-189, 2000.

4. Bork P, Gibson TJ, Applying motif and profile searches, Methods Enzymol 266:162-184, 1996.

5. Bork P, Koonin EV, Predicting functions from protein sequences-where are the bottlenecks? Nat Genet 18:313-318, 1998.

6. Wong WC, Maurer-Stroh S, Eisenhaber F, More than 1,001 problems with protein domain databases: Transmembrane regions, signal peptides and the issue of sequence homology, PLoS Comput Biol 6:e1000867, 2010. 
7. Eisenhaber F, A decade after the first full human genome sequencing: When will we understand our own genome? J Bioinform Comput Biol 10:1271001, 2012.

8. Sirota FL, Batagov A, Schneider G, Eisenhaber B, Eisenhaber F, Maurer-Stroh S, Beware of moving targets: Reference proteome content fluctuates substantially over the years, J Bioinform Comput Biol 10:1250020, 2012.

9. Kuznetsov V, Lee HK, Maurer-Stroh S, Molnar MJ, Pongor S, Eisenhaber B, Eisenhaber F, How bioinformatics influences health informatics: Usage of biomolecular sequences, expression profiles and automated microscopic image analyses for clinical needs and public health, Health Information Science and Systems 1:2, 2013. 